\title{
ON THE INVARIANT MOMENTUM HYPOTHESIS AT EXTREMELY LARGE VELOCITIES
}

\author{
V. Baranauskas, Faculdade de Engenharia Elétrica e Computação, Universidade Estadual de \\ Campinas, Av. Albert Einstein N. 400, 13083-970 - Campinas - SP - Brasil
}

\begin{abstract}
Starting from the hypothesis of the invariancy of the subluminal momentum and applying the beyond-Lorentz transformations we derive a new equation for the invariant momentum-velocity of particles which can be applied to subluminal or superluminal particles. The kinetic energy shows a maximum at the velocity of light and falls at subluminal or superluminal velocities. There is good agreement with the Newtonian and relativistic results for particle momentum and energy at subluminal velocities.
\end{abstract}

\section{INTRODUCTION}

The Special Theory of Relativity Theory (STR) makes use of the Lorentz transformations, whose real solutions are limited to velocities below the velocity of light (subluminal velocities). One of the STR's main assertions is that the relativistic momentum is conserved regardless of the frame of reference. The mass of a particle assumes an energy content and apparently increases with an increase in its velocity, becoming infinite at the velocity of light (c). Photons or neutrinos are thus considered particles with zero rest-mass since that they can travel with limited energies at the velocity of light. Recent experiments, however, suggest that neutrinos are massive particles [1].

When exploring the extreme limits of bunches of particles, why not to seek more general transformations that can also be applied above $\mathrm{c}$ (superluminal velocities), including the Lorentz transformation as a special case? Recently, we demonstrated the existence of such general transformations which also obey the principle of the constancy of c [2].

In the present work, starting from the hypothesis of invariant momentum and applying such general transformations, we demonstrate the existence of a new equation for the momentum that agrees with the STR's predictions for momentum of subluminal phenomena but can also be applied at superluminal velocities. The

This work is dedicated to the memory of my son, V. B. Filho (19871997). The author acknowledges the kind help of S. F. Durrant in editing the manuscript and CNPq, CAPES, FAPESP and the PAC-99 organizers for partial financial support.

Email: vitor@dsif.fee.unicamp.br kinetic energy of the particle, calculated from the integration of this new momentum-velocity equation, shows that all terms depend on the velocity, which contradicts the STR's prediction of the existence of an associated rest energy related only to the mass of the particle. Finally, predictions are made concerning the dependence of the momentum and kinetic energy on the velocity of superluminal particles.

\section{BEYOND-LORENTZ TRANSFORMATIONS}

Accepting the hypothesis that the velocity of light is constant, i. e., does not depend on the reference system, we can write the following transformation equations between a system reference $S$ and a system $S$ ' that moves with a constant speed $\mathrm{v}$ in the direction of the axis $\mathrm{X}$, relative to $\mathrm{S}$ :

$$
\begin{gathered}
\mathrm{x} \longrightarrow \mathrm{x}^{\prime}=\beta_{\mathrm{B}} \mathrm{x}-\sqrt{\beta_{\mathrm{B}}^{2}-1} \mathrm{ct} \\
\text { and } \mathrm{t} \longrightarrow \mathrm{t}^{\prime}=\beta_{\mathrm{B}} \mathrm{t}-\sqrt{\beta_{\mathrm{B}}^{2}-1} \frac{\mathrm{x}}{\mathrm{c}} \\
\text { with } \quad \beta_{\mathrm{B}}=\sqrt{\sum_{\mathrm{n}=0}^{\mathrm{k}}\left(\frac{\mathrm{v}}{\mathrm{c}}\right)^{2 \mathrm{n}}}=\sqrt{\frac{\left(\frac{\mathrm{v}}{\mathrm{c}}\right)^{2(\mathrm{k}+1)}-1}{\left(\frac{\mathrm{v}}{\mathrm{c}}\right)^{2}-1}}
\end{gathered}
$$$$
\text { with }
$$

These equations can be applied to describe light wave propagation invariance under uniform translation of $S$ ' relative to $S$ in the $x$ direction for any value of the order number $\mathrm{k}(\mathrm{k}=1,2, \ldots)$. It can easily be verified for subluminal velocities that the above equations can be made as close as is wanted to the Lorentz transformations by a convenient choice of $\mathrm{k}$ ( $\mathrm{k}$ large). However, unlike with the Lorentz transformations, the new solutions are always real for any velocity, including superluminal velocities.

\section{MOMENTUM-VELOCITY RELATIONSHIP}

Through the conservation of the energy-momentum in 
particle collisions it was demonstrated that the relativistic momentum can be expressed by [3]:

$$
\mathrm{p}=\mathrm{m} \frac{\Delta \mathrm{x}}{\Delta \mathrm{t}^{\prime}}
$$

which, with application of the beyond-Lorentz transformations can be written as:

$$
\mathrm{p}=\frac{\mathrm{m} \mathrm{v}}{\left|\beta_{\mathrm{B}}-\frac{\mathrm{v}}{\mathrm{c}} \sqrt{\beta_{\mathrm{B}}^{2}-1}\right|}=\gamma_{\mathrm{B}} \mathrm{m} \mathrm{v}
$$

where $\gamma_{\mathrm{B}}$ is the new correction factor. This equation can be applied for any velocity, either subluminal or superluminal. Figure 1 compares the curves of momentum versus velocity calculated from this result (equation (4)) and the STR momentum, respectively. Newtonian momentum is also ploted for reference.

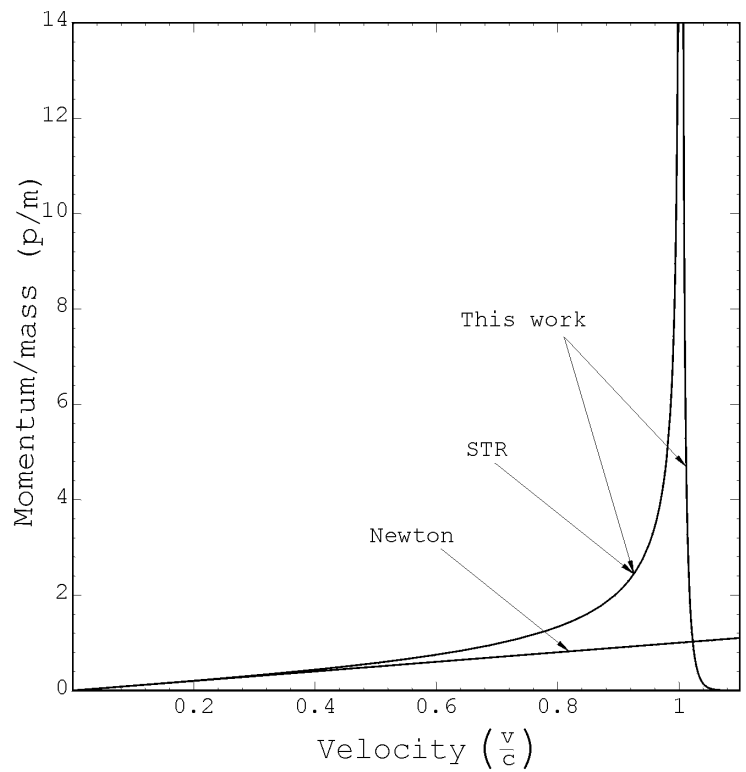

Figure 1: Momentum versus velocity: Comparison of the results of the Newtonian, STR and the present approach.

We observe a good agreement between this new result and the STR momentum for subluminal velocities. Thus, great part of the subluminal experiments that validate the relativistic momentum also confirm the new momentum equation. However, this new equation can also be used to foresee the momentum at superluminal velocities. As we can see, when a particle is passing the light velocity barrier its momentum is strongly attenuated, as in the evanescent transmission of particles-waves in tunneling phenomena. The existence of a very thin spike at $\mathrm{v}=\mathrm{c}$, with an upwards divergence at $\mathrm{c}$ - and a strong attenuation at $\mathrm{c}+$ suggests a very high momentum for luminal particles, but not a limitless momentum. It should also be observed that in spite of the momentum tending to zero for superluminal velocities, the velocity of the particle is not reduced since its momentum-velocity relationship differs both from Newtonian and the usual relativistic formulas.

\section{KINETIC ENERGY}

We can calculate the kinetic energy from the usual expression of the integration of a force $\mathrm{F}$ continuously acting on a particle along the axis $\mathrm{x}$ :

$$
\mathrm{K}=\int \mathrm{F} d \mathrm{x}=\int \mathrm{dp} \frac{\mathrm{dx}}{\mathrm{dt}}=\mathrm{p} \mathrm{v}-\int_{\mathrm{v}=0}^{\mathrm{v}} \mathrm{pdv}
$$

and applying equation (4), we find

$$
\mathrm{K}=\mathrm{m} \mathrm{c}^{2}\left(\Phi_{1}(\mathrm{v})-\Phi_{2}(\mathrm{v})\right)
$$

with

and

$$
\begin{gathered}
\Phi_{1}(\mathrm{v})=\gamma_{\mathrm{B}} \frac{\mathrm{v}^{2}}{\mathrm{c}^{2}} \\
\Phi_{2}(\mathrm{v})=\int_{0}^{\mathrm{v}} \gamma_{\mathrm{B}} \frac{\mathrm{v}}{\mathrm{c}^{2}} \mathrm{dv}
\end{gathered}
$$

We did not find an algebraic solution to solve the integral for $\Phi_{2}(\mathrm{v})$ but it can easily be numerically integrated. The curve of the kinetic energy versus the velocity of the particle shows a maximum at the velocity of light which decays at subluminal or superluminal velocities (see Figure 2 (a)). Again, there is a good agreement with the STR predictions for subluminal velocities. Besides this good fitting, it should be pointed out that there is a fundamental difference between the usual relativistic formula for kinetic energy $\left(\mathrm{K}=\mathrm{m} \mathrm{c}^{2}\left(\left(1-\mathrm{v}^{2} / \mathrm{c}^{2}\right)^{-1 / 2}-1\right)\right)$ and the formula presented by equation (6) since all terms ( $\Phi_{1}$ and $\Phi_{2}$ ) in the latter depend on $\mathrm{v}$. Thus, it is demonstrated that the hypothesis of an energy associated to the mass of the particle in rest in not a necessary condition for obtaining the results foreseen by STR. The mass of the particle can be assumed invariant with the velocity, being just a multiplying parameter of the related energy.

For low velocities (see Figure 2 (b)) the functions $\Phi_{1}$ and $\Phi_{2}$ may be approximated respectively by $\Phi_{1}(\mathrm{v}) \cong \frac{\mathrm{v}^{2}}{\mathrm{c}^{2}}$ and $\Phi_{2}(\mathrm{v}) \cong \frac{1}{2} \frac{\mathrm{v}^{2}}{\mathrm{c}^{2}}$. Therefore equation

(6) is reduced to $\mathrm{K} \cong \frac{1}{2} \mathrm{~m} \mathrm{v}^{2}$, which coincides with the Newtonian formula.

For subluminal velocities very close to the velocity of light $\left(\mathrm{v} \cong \mathrm{c}^{-}\right)$we have that $\Phi_{1} \cong v_{\mathrm{B}} \approx\left(1-\mathrm{v}^{2} / \mathrm{c}^{2}\right)^{-1 / 2}$ and $\Phi_{2} \cong 1$ (see Figure 2 (a)). In this case, equation (6) reduces to the usual relativistic formula for kinetic energy: $\mathrm{K} \cong \mathrm{m} \mathrm{c}^{2}\left(\left(1-\mathrm{v}^{2} / \mathrm{c}^{2}\right)^{-1 / 2}-1\right)$. 


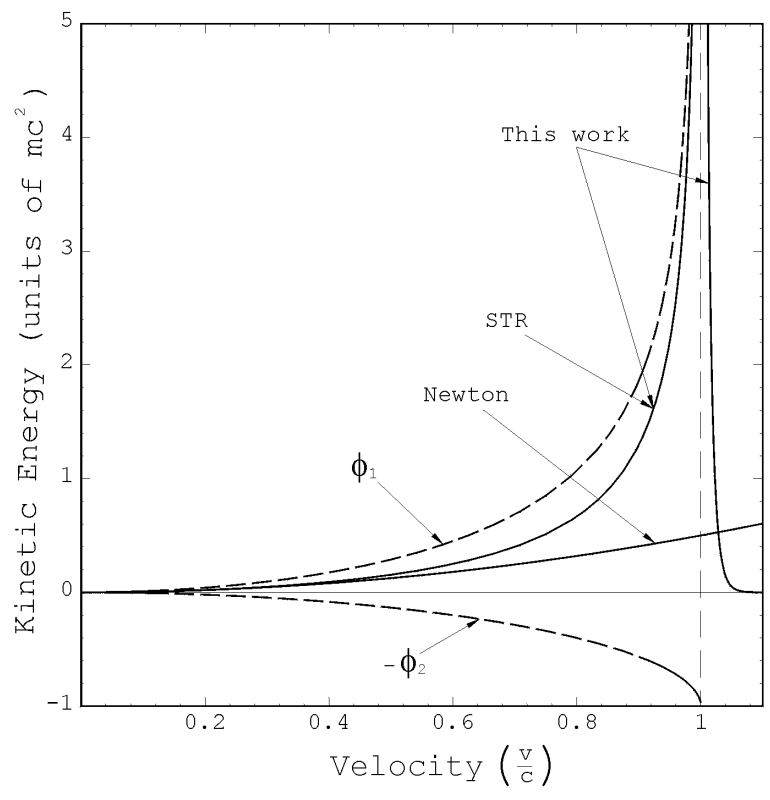

(a)

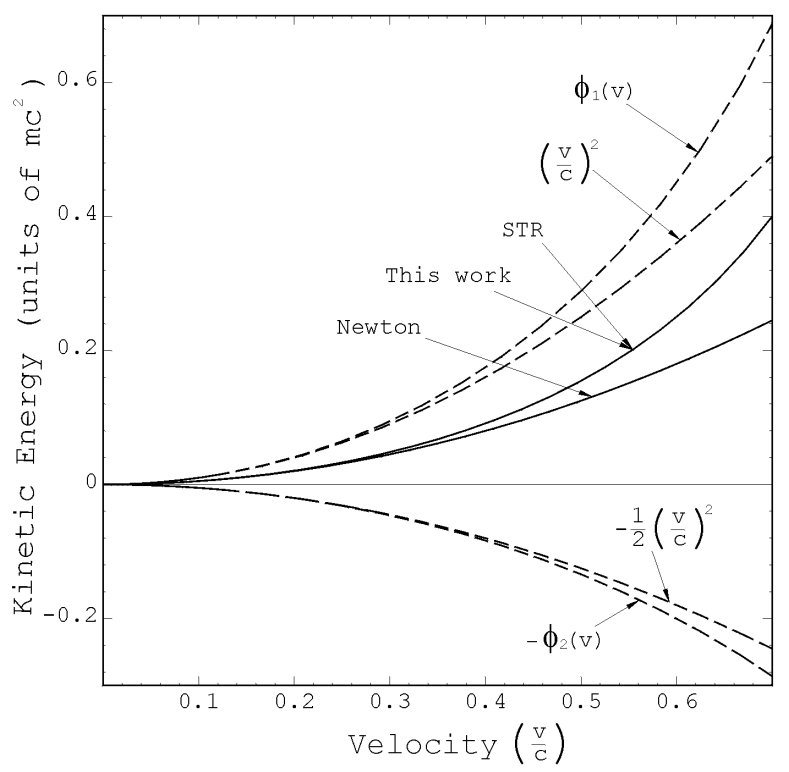

(b)

Figure 2: (a) Kinetic energy versus velocity: Comparison of Newtonian, STR, and of this approach. The dependence of the functions $\Phi_{1}$ and $\Phi_{2}$ on velocity are also shown. For subluminal velocities there is a good agreement between this work and the STR, however, there are no terms independent of the velocity.

(b) For low velocities, $\Phi_{1}$ and $\Phi_{2}$ approaches a dependence on $\frac{\mathrm{v}^{2}}{\mathrm{c}^{2}}$ and $\frac{1}{2} \frac{\mathrm{v}^{2}}{\mathrm{c}^{2}}$, respectively.

Surpassing the barrier of the velocity of light the kinetic energy of the particle diminishes, as illustrated in Figure 2 (a). The fall in the kinetic energy with an increase in the velocity is an interesting effect because it is not necessary to increase the energy of the particle to increases its velocity. On the contrary, the particle needs to lose energy (by radiation?) to increase its velocity. The vacuum for superluminal particles behaves as a superfluid. If the superluminal particle emits light to lose kinetic energy the emitted photons may be luminal, thus, not changing the refractive index of the vacuum. The radiation emitted by the superluminal particle may be similar to the Cerenkov radiation. In fact, the new equations presented here may be rewritten for the propagation of particles in a material medium other than vacuum (refractive index $>1$ ), thus serving as an explanation for the Cerenkov radiation in liquid and solid materials.

Just as in the dynamics of a resonance problem, the value of the energy of the luminal particle should be very high due to the singularity at $\mathrm{v}=\mathrm{c}$, but not infinite. Other conditions are necessary to calculate its limit. Equation (6) explicitly shows that the kinetic energy depends directly on the mass of the particle, thus particles with larger mass will have to have larger energies to become luminal particles. We did not find any support in our derivation for the supposition that luminal particles have to have zero mass.

\section{CONCLUSIONS}

Through the use of the beyond-Lorentz transformations (velocity of light is constant) we derived a new equation for the invariant momentum-velocity of particles that can be applied to subluminal or superluminal particles. Good agreement of this new equation was demonstrated (for subluminal velocities) with the relativistic momentum and with Newtonian momentum, in its respective range of application. For superluminal particles this new equation foresees that the momentum is strongly attenuated with an increase of velocity. The kinetic energy calculated using this new equation for the momentum shows a maximum at the velocity of light and decays at subluminal or superluminal velocities. There is also a good agreement with the relativistic and Newtonian results for subluminal velocities. However, there is no need to consider an energy associated with the rest-mass of the particle to calculate its kinetic energy.

\section{REFERENCES}

[1] D. Normile, Science, 280, p. 1689 (1998).

[2] V. Baranauskas in 6th European Particle Accelerator Conference EPAC-98, 1998, edited by S. Myers, L. Liljeby, Ch. Petit-Jean-Genaz, J. Poole, K.-G. Rensfelt, (Institute of Physics Publishing, Bristol, 1998), p. 835 .

[3] G. N. Lewis and R. Tolman, Phil. Mag. 18, p. 510 (1909). 